\title{
Childhood Cat Bites Relate to Increased Adulthood Severity of Schizotypy, Psychotic-Like Experiences, and Social Anhedonia in a Transdiagnostic Psychiatric Sample
}

\author{
Jeffrey S. Bedwell ${ }^{\mathrm{a}}$ Christopher C. Spencer ${ }^{\mathrm{a}} \quad$ Amber L. Southwell $^{\mathrm{b}}$ \\ a Department of Psychology, University of Central Florida, Orlando, FL, USA; \\ ${ }^{b}$ Burnett School of Biomedical Sciences, University of Central Florida, Orlando, FL, USA
}

\section{Keywords}

Cat $\cdot$ Schizophrenia $\cdot$ Schizotypy $\cdot$ Depression $\cdot$ Bipolar disorder · Pasteurella multocida $\cdot$ Bartonella henselae .

Toxoplasma gondii · Positive symptoms · Psychotic-like experiences · Negative symptoms · Anhedonia

\begin{abstract}
Introduction: Previous research has linked childhood cat scratches and bites to an increased risk for depression, and childhood cat ownership to increased risk of schizophrenia, bipolar disorder, and social anhedonia. Our group previously reported that childhood cat bites, but not ownership, related to increased schizotypy severity in an undergraduate sample. Methods: The current study expands this research by inquiring about cat bites and ownership in a transdiagnostic adult sample $(N=162 ; 51 \%$ female; mean age $=38.15$, $\mathrm{SD}=10.65)$, composed of schizophrenia spectrum disorders $(n=30)$, bipolar disorders $(n=30)$, unipolar depression $(n=$ $23)$, and nonpsychiatric participants $(n=79)$. Participants completed a diagnostic interview, scales of symptom severity, and a cat interaction history interview. Results: Across the entire sample, self-report of cat bites prior to age 13, but not after, related to greater current severity of overall schizo-
\end{abstract}

typy, self-reported and clinician-rated psychotic-like symptoms, and social anhedonia, when compared to individuals who reported no lifetime cat bites. Cat bites prior to age 13 did not relate to severity of depression, non-social anhedonia, or clinician-rated negative symptoms. Self-report of residing with a cat prior to age 13 , or a first cat bite after age 12 , did not relate to any symptom severity measure examined. Conclusions: One theory for these findings is that an unknown infectious agent common in cat saliva interacted with brain development in childhood to increase the likelihood of these symptoms. A novel theory for the infectious agent Pasteurella multocida is discussed. Future research can examine candidate infectious agents to identify potential causal mechanisms for these relationships.

C 2020 S. Karger AG, Basel

\section{Introduction}

In a previous publication [1], we reported that undergraduate students who endorsed experiencing a cat bite that pierced the skin prior to age 13 had a higher score on a self-report measure of schizotypy - a personality structure related to the schizophrenia spectrum [2]. This rela-

\section{KARGER}

(c) 2020 S. Karger AG, Basel

karger@karger.com

www.karger.com/psp
Assoc. Prof. Jeffrey S. Bedwell, PhD 
tionship was found both with the total schizotypy score and with the disorganized symptom factor score [1]. Our speculative theory was that a childhood infection from the cat bite may have caused chronic brain inflammation or changes in development which then caused or increased schizotypy symptoms. In the current manuscript, we explore this finding by asking about a lifetime history of cat bites in a transdiagnostic psychiatric sample from the community. A previous history of cat scratches $[3,4]$ or bites [5] has primarily been associated with depression. The link between scratches and depression was specific to cats and not found with dog bites in one study that examined both [4]. One study found that when entering both cat scratches and bites along with other variables in the same model, only cat scratches related to increased depression severity [3]. A different study examined electronic health records for a diagnosis of depression and animal injuries in adulthood in a large sample $(n=$ $1,327,368)[5]$. The rate of depression was highest for individuals with a history of cat bites ( 41.3 vs. $8.8 \%$ for the whole sample). The rate of depression in individuals with cat bites was statistically greater than the rate found for a bite or injury from any other animal (28.5\%) or dog bites in particular (28.3\%); however, both of these categories were significantly greater than the overall sample rate. The study did not examine depression diagnoses or animal injuries that were entered into the medical record prior to age 18. It appears that only one study has examined the association of cat scratches with the diagnosis of schizophrenia, finding no association in a large sample using cross-sectional design [4]. To the authors' knowledge, none have specifically examined how cat bites relate to schizophrenia.

Several studies have linked childhood cat ownership to increased risk for severe mental illness such as schizophrenia and bipolar disorder, potentially through infection from Toxoplasma gondii (i.e., T. gondii; toxoplasmosis; for review see [6]). For example, one study found that the IgG antibody to T. gondii was significantly higher in individuals diagnosed with schizophrenia as compared to depressive/anxiety disorders or no disorder [7]. However, when these authors statistically accounted for residing with a cat in childhood, the increased incidence of toxoplasmosis in schizophrenia participants was no longer significant, suggesting that toxoplasmosis was a secondary marker of contact with cats. This implies that a different mechanism related to residing with cats in childhood has a more direct causal role for later development of schizophrenia, which may include a different infectious agent frequently carried by cats. A large cross-sectional study of Finnish adults found that after covarying for a cat ownership variable of "never, previous, or current," toxoplasmosis related to increased self-report of lifetime psychotic-like symptoms, but not the diagnosis of schizophrenia [8]. Another study found that when accounting for the T. gondii exposure occurring prior to schizophrenia onset, the incidence rate ratio for the relationship was 2.78 , but the study did not examine interactions with childhood cat ownership [9]. However, other studies which did not examine T. gondii reported that childhood cat ownership did not relate to schizophrenia $[4,8]$, unipolar depression [4], bipolar disorder [4], psychotic experiences in adolescents [10], or schizotypy severity in young adults [1]. A study from the Northern Finland Birth Cohort 1966 found that adults who self-reported cat ownership prior to age 7 endorsed a greater level of social, but not physical, anhedonia severity [11]. This study also found that, after covarying for potential confounding variables, there were no statistically significant relationships between owning a cat prior to age 7 and the diagnosis of schizophrenia, or scores on scales of perceptual aberrations and schizoid traits.

Rather than examine the relationship between cat history variables and diagnostic categories, we focused on cat history relationships in relation to dimensional scales of specific types of psychiatric symptom severity across a transdiagnostic psychiatric sample. This approach is consistent with recommendations from the Research Domain Criteria (RDoC) initiative of the National Institute of Mental Health $[12,13]$. Based on the existing literature, we included self-report scales of schizotypy and subtypes of anhedonia, along with clinician-rated severity of positive and negative schizophrenia spectrum symptoms and depression. Based on our initial finding in a nonpsychiatric sample [1], we also hypothesized that report of a cat bite prior to age 13 would relate to increased severity of overall schizotypy, with the largest effect size found for disorganized symptoms. We hypothesized that endorsing a lifetime history of cat bites would relate to increased severity of recent depression [5]. Finally, based on a recent study [11], we hypothesized that childhood cat ownership would relate to increased social, but not non-social, anhedonia.

\section{Materials and Methods}

\section{Participants}

This study was approved by the Institutional Review Board of the University of Central Florida and follows the ethical guidelines described in the Declaration of Helsinki. The primary aim of the 
Table 1. Demographic and diagnostic characteristics of the sample

\begin{tabular}{|c|c|c|c|c|c|c|c|}
\hline & $n$ & Female sex & Age, years & White & $\begin{array}{l}\text { Hispanic/ } \\
\text { Latinx }\end{array}$ & $\begin{array}{l}\text { Years of } \\
\text { education }\end{array}$ & 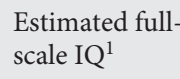 \\
\hline All participants & 162 & $51.2 \%$ & $38.15(10.65)$ & $63.6 \%$ & $18.5 \%$ & $14.52(2.17)$ & $100.95(10.06)$ \\
\hline Schizophrenia spectrum disorder & 30 & $50.0 \%$ & $39.00(10.27)$ & $50.0 \%$ & $13.3 \%$ & $13.47(1.66)$ & $100.90(10.68)$ \\
\hline Schizophrenia & 18 & & & & & & \\
\hline Schizoaffective disorder & 6 & & & & & & \\
\hline Paranoid personality disorder & 1 & & & & & & \\
\hline Bipolar disorders & 30 & $63.3 \%$ & $38.83(10.32)$ & $90.0 \%$ & $16.7 \%$ & $15.53(2.58)$ & $101.07(9.53)$ \\
\hline Bipolar I disorder, nonpsychotic & 20 & & & & & & \\
\hline Bipolar I disorder, with psychosis & 7 & & & & & & \\
\hline Bipolar disorder not otherwise specified & 3 & & & & & & \\
\hline Unipolar depression & 23 & $52.2 \%$ & $39.74(10.65)$ & $65.2 \%$ & $26.1 \%$ & $12.82(1.73)$ & $97.22(12.92)$ \\
\hline
\end{tabular}

Cell values are listed in the format: mean (standard deviation) unless otherwise stated. ${ }^{1}$ Standard score from Wide Range Achievement Test -4 th edition - Reading subtest.

larger funded study was to examine EEG during visual processing. However, we included questions about cat interaction history due to our ongoing interest in this topic from our previous publication [1]. Participants were recruited for the overarching study using two different types of advertisements. One mentioned that we were looking for individuals with a diagnosis of schizophrenia, schizoaffective disorder, or bipolar disorder, while the other mentioned that we were looking for adults between the ages of 21 and 55 (without mentioning any diagnoses). These advertisements were placed in a variety of locations including local psychiatric facilities, local newspapers, and the Craigslist website.

Although we listed three diagnoses of primary interest on the flyers, the study used a transdiagnostic symptom severity approach, which included any psychiatric diagnosis (unless listed in exclusion criteria below) or the absence of a psychiatric diagnosis. Inclusion criteria also included being between the ages of 21 and 55. Exclusion criteria included: history of a neurological disorder, seizures, or head injury resulting in loss of consciousness for more than $15 \mathrm{~min}$, substance abuse or dependence in the last 6 months (per diagnostic interview), heavy alcohol use or any recreational drug use in the $72 \mathrm{~h}$ prior to the session (per self-report), estimated full scale IQ $<70$, visual acuity worse than 20/50 (allowing for corrective lenses), and indication of color blindness. Following a phone screening, eligible participants were invited to the first of two sessions. Participants were provided a monetary stipend at the end of each session. The second session included EEG analysis of visual processing which has been published previously [14]; however, that publication did not include any data regarding cat interaction history.

Following recruitment attempts, we assessed 175 participants in the first session. Of these, 13 were not eligible for use in the final analyses for the following reasons: substance dependence or abuse during past 6 months $(n=8)$, visual acuity worse than $20 / 50(n=$
$3)$, color blindness $(n=1)$, and estimated full-scale IQ $<70(n=1$; estimated from standard score on the Wide Range Achievement Test -4 th edition [15] Reading subtest). Although the four individuals excluded for visual issues would have been useful for the current analyses, those participants were excluded early in the session and did not complete a diagnostic interview or symptom measures.

Analyses examined the remaining transdiagnostic sample of 162 adults from the community. Of the 162 participants, 79 (48.8\%) had no current psychiatric disorder, 30 (18.5\%) had a schizophrenia spectrum disorder, 30 (18.5\%) had a bipolar disorder, and 23 (14.2\%) had unipolar depression. These four groups did not statistically differ in age, sex, race, or Hispanic/Latinx ethnicity (all $p>$ 0.17 ). See Table 1 for list of specific diagnoses and demographic characteristics. As Table 1 only lists percent White/Caucasian for race, independent of Hispanic/Latinx ethnicity, the sample was 63.6\% $(n=103)$ White/Caucasian, 22.8\% $(n=37)$ Black/AfricanAmerican, 3.1\% $(n=5)$ Asian, and 10.5\% $(n=17)$ endorsed "more than one race."

\section{Procedures}

Following informed consent, participants completed the selfreport Schizotypal Personality Questionnaire - Brief Revised (SPQ-BR; [2]). The SPQ-BR includes 32 items reflecting seven subordinate and four superordinate factors [16]: Cognitive-Perceptual (Unusual Perceptual Experiences, Odd Beliefs, Ideas of Reference/Suspiciousness), Interpersonal (Constricted Affect/No Close Friends), Disorganized (Odd/Eccentric Behavior and Odd Speech), and Social Anxiety (Excessive Social Anxiety). The response set is a 5-point Likert scale ranging from "Strongly Disagree" to "Strongly Agree." Participants also completed two scales that assess trait anhedonia (i.e., difficulty anticipating or experiencing pleasure). The first primarily assessed trait non-social an- 
hedonia, the Temporal Experience of Pleasure Scales (TEPS), with 18-items and a 6-point Likert scale ranging from "Very False for $\mathrm{Me}$ " to "Very True for Me" [17]. This scale produces separate scores for anticipatory (TEPS-A) and consummatory (TEPS-C) anhedonia. The second scale assessed trait social anhedonia, the Anticipatory and Consummatory Interpersonal Pleasure Scale (ACIPS) [18], with 17 items and the same response scale as the TEPS. Unlike the TEPS, factor analysis has not supported separate factors for anticipatory and consummatory anhedonia on the ACIPS [18]; therefore, the total score was used for analyses. Increased anhedonia is indicated by lower scores on both the TEPS and ACIPS.

A trained clinical psychology doctoral student administered the Structured Clinical Interview for DSM-IV Axis I Disorders [19] and the schizotypal, paranoid, and avoidant personality disorder (i.e., schizophrenia spectrum) sections of the Structured Clinical Interview for DSM-IV Axis II Disorders [20]. Final diagnoses were determined through case presentations with a licensed psychologist (author J.S.B.). Participants also completed the Structured Clinical Interview for the Positive and Negative Syndrome Scale (PANSS) [21, 22], which allows for clinician ratings of symptom severity on 30 items using a 7-point Likert scale ranging from "Absent" to "Extreme." The PANSS produces a Positive Scale score (e.g., delusions, hallucinations), a Negative Scale score (e.g., blunted affect. emotional withdrawal), and a General Psychopathology Scale score (e.g., anxiety, depression). As the hypotheses for this study involved schizophrenia spectrum symptoms, the Positive Scale and Negative Scale scores were used in analyses. In addition, to address the hypothesis regarding depression, the Depression item severity score from the General Psychopathology Scale (item G6) was also analyzed.

Finally, among a larger battery of measures, the cat questions were provided in an interview format with queries regarding details: (1) "Prior to age 13, did you have any cats in the place where you resided? Around what age(s)" and (2) "Have you ever been bitten by a cat? Did it pierce your skin? Around what age(s)?" We asked the second question based on our earlier findings [1] but broadened the question to include bites at any age. In addition to being consistent with the age cutoff from our earlier paper, asking about a history prior to age 13 is used by most $[4,6,7]$, although not all [11], of the studies by others on this topic.

\section{Statistical Analyses}

Four participants were excluded from cat bite analyses, and four different participants were excluded from residing with cat analyses, for unclear or missing information about the related age. We only considered bites in analyses that were reported to have pierced the skin. In order to compare findings to our previous report, for cat bites, we created a categorical variable of never, yes prior to age 13, and yes after age 12 . For the question on cats in household, we only asked about prior to age 13 , so that variable remained binary (i.e., yes/no). We examined each of these cat history questions as dependent variables in separate logistic regressions using IBM SPSS Statistics (version 25). Due to the small number of cases endorsing a cat bite in each age range, we were not able to reliably examine categorical predictors, including demographic (e.g., sex) and diagnosis variables, as this comparison resulted in many cells with less than 10 (and often less than 5) participants. While this was not as problematic with the binary residing with a cat variable, we wanted to be consistent with analyses across the two cat history variables. Therefore, we restricted all analyses of predictors to continuous variables, which ensured that there was always at least 10 participants within each cell of either categorical dependent variable.

As the cat bite variable had three categories, a multinomial logistic regression was used, while a binary logistic regression was used for the residing with a cat variable. For the multinomial logistic regression, the omnibus $\chi^{2}$ test for is provided for each predictor from likelihood ratio tests. Due to a priori hypotheses about schizotypy factor score findings being selective to participants reporting a first bite prior to age 13, each of the two age groups reporting a cat bite were statistically compared to the group reporting no lifetime cat bites.

The symptom severity scores were examined for statistical outliers as defined by a $Z$-score of \pm 3.00 compared with the entire sample. There were no statistical outliers for the four SPQ-BR factor scores or total score. For the anhedonia scales, there was 1 participant who was an outlier across all three scales and 2 who were outliers for just the TEPS-C. For the PANSS, there were 3 participants who were outliers for the Positive Scale, 3 different participants for the Negative Scale, and no outliers were found for the Depression Item score. Across all anhedonia and PANSS scales, all outliers were in the direction of reporting greater severity of symptoms. The scores for the respective scales and participants were then removed for the remainder of analyses. Following removal of outliers, skewness and kurtosis for the predictor sets were: SPQ-BR factor and total scores (skewness $< \pm 0.34$, kurtosis $< \pm 1.05$ ), anhedonia scales (skewness $< \pm 0.87$, kurtosis $< \pm 0.43$ ), and PANSS scales (skewness $< \pm 1.48$, kurtosis $< \pm 1.82$ ).

For each set of predictors, forced simultaneous entry was used. The first set had no missing values and included current age, estimated IQ, and years of education (zero-order $r<0.36$ among predictors). The second set of predictors was the four schizotypy factor scores from the SPQ-BR, which also had no missing values $(r<0.67$ among predictors). The SPQ-BR total score was entered alone in a separate regression. The third set of predictors was the three anhedonia scale scores (TEPS-A, TEPS$\mathrm{C}$, and ACIPS total score; $r<0.63$ among predictors). One participant was missing all three anhedonia scores and three were removed across the predictor set for being statistical outliers. Finally, the last set of predictors was from the PANSS clinician interview of symptom severity (Positive Scale score, Negative Scale score, and the Depression Item score; $r<0.45$ among predictors). Only 143 participants (88.3\% of sample) had available PANSS scores, as those participants did not complete the interview during the first session and did not return for a second session. Using a binary logistic regression, the 19 participants who were missing the PANSS did not differ statistically from the remainder of the sample in terms of age, sex, race, Hispanic/Latinx ethnicity, years of education, estimated IQ, history of psychosis, or diagnostic class (all $p$ values $>0.07$ ), or either of the cat history variables (both $p$ values $>0.11$ ). As noted above, 6 participants who completed the PANSS were removed from the predictor set after being defined as statistical outliers.

All data used for the final analyses and for tables and figures is publicly available in an online repository on the "figshare" website: https://doi.org/10.6084/m9.figshare.9764567.v3. 
Table 2. Descriptive statistics and multinomial logistic regression results related to cat bite history

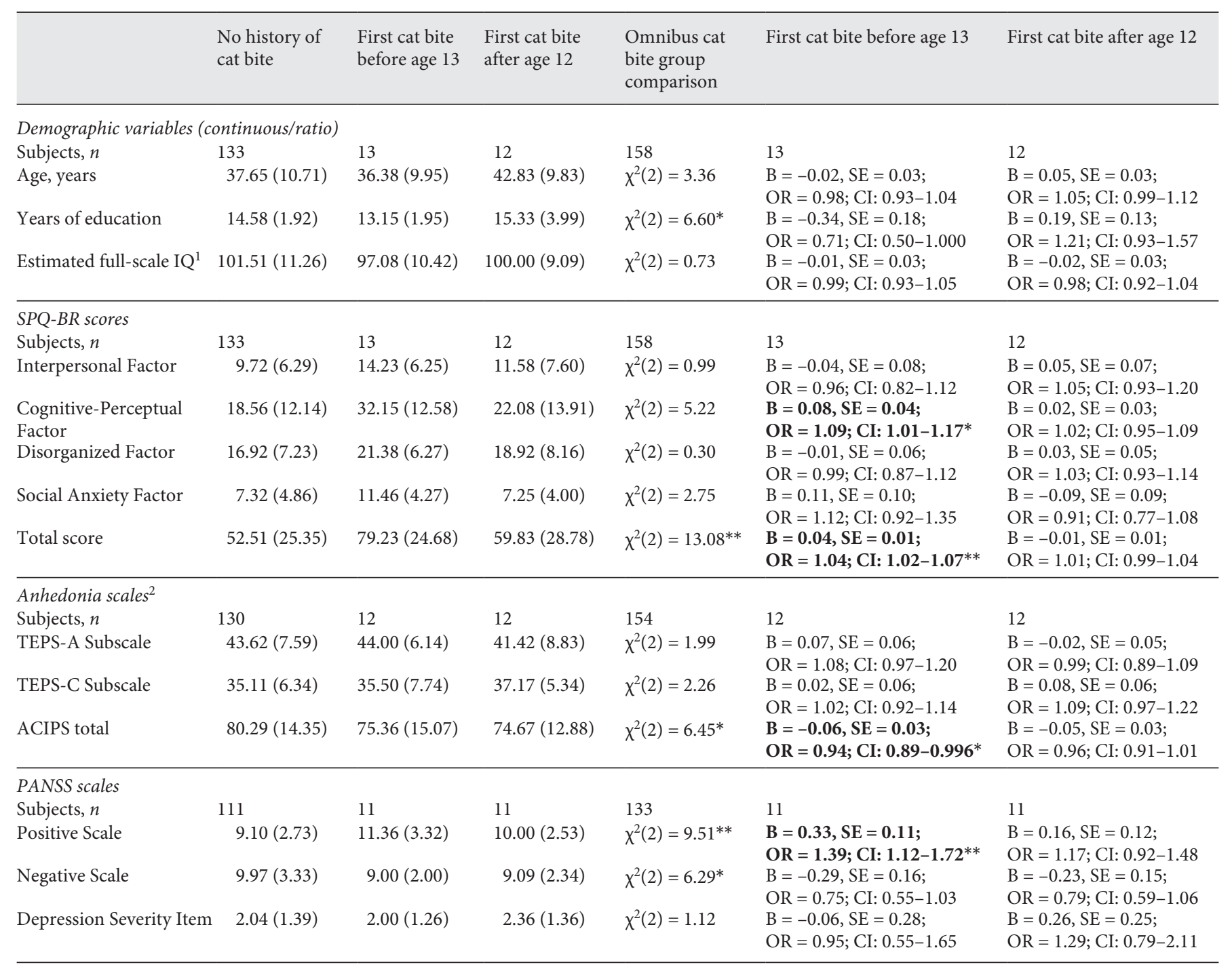

Cell values in first three columns are listed in the format: mean (standard deviation). Four of the 162 participants did not have age of bite available. B, unstandardized beta; SE, standard error for beta; OR, odds ratio; CI, $95 \%$ confidence interval for odds ratio. ${ }^{*} p<0.05$; ${ }^{* *} p<0.01,{ }^{* * *} p<0.001$ across the three groups (for omnibus test) or in comparison with participants reporting no lifetime cat bites for last two columns. SPQ-BR, Schizotypal Personality Questionnaire - Brief Revised; TEPS-A, Anticipatory subscale of the Temporal Experience of Pleasure Scale; TEPS-C, Consummatory subscale of the Temporal Experience of Pleasure Scale; ACIPS, Total score from the Anticipatory and Consummatory Interpersonal Pleasure Scale; PANSS, Positive and Negative Syndrome Scale. ${ }^{1}$ Full-scale IQ estimated using the standardized score from the Reading Subtest of the Wide Range Achievement Test - 4th edition. ${ }^{2}$ For all anhedonia scales, lower scores indicate greater anhedonia (i.e., less pleasure).

\section{Results}

\section{Relationship between the Two Categorical Cat History Variables}

Of participants with available data for both cat bites and residing with a cat $(n=154)$, for the 56 participants who reported residing with a cat prior to age $13,19.6 \%$ $(n=11)$ reported being bitten before age 13 and $10.7 \%$ $(n=6)$ reported being bitten after age 12. In contrast, for the 98 participants reporting that they did not reside with a cat prior to age $13,2.0 \%(n=2)$ reported being bitten prior to age 13 , and $6.1 \%(n=6)$ reported being bitten after age 12 . Therefore, there was a statistically significant relationship between the two cat history variables, $\chi^{2}(2)=$ $16.14, p<0.001$. 
Fig. 1. Cat bite history in relation to selfreported schizotypy severity.

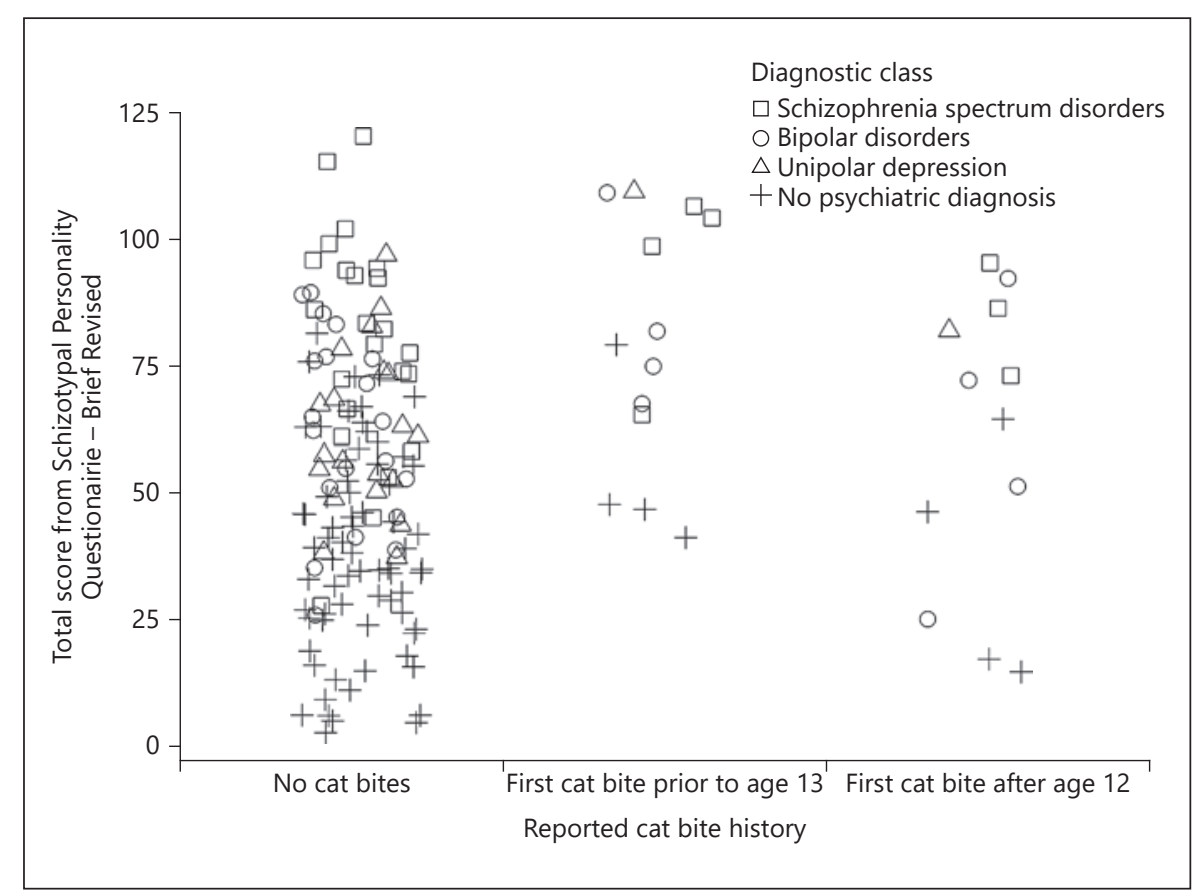

\section{Relationships with Cat Bites}

See Table 2 for descriptive and inferential statistics for all analyses involving the cat bite variable. In a multinomial logistic regression, the dependent variable of cat bite category did not relate to current age and estimated IQ. While the omnibus comparison across the three groups was statistically significant for years of education, neither of the bite age groups differed from participants who reported no bite.

The regression with the SPQ-BR total score revealed a statistically significant relationship with the cat bite variable, as participants reporting a bite prior to age 13 , but not after, reported greater overall schizotypy compared to the group who reported no lifetime cat bites (Fig. 1). The regression with the four SPQ-BR schizotypy factor scores showed that only the Cognitive-Perceptual factor was significantly related to reported cat bites, with only individuals reporting a bite prior to age 13 having a higher score than participants reporting that they had never been bitten. The analysis with the three anhedonia scales found that participants reporting the first bite prior to age 13, but not after, reported more severe social anhedonia (i.e., lower ACIPS score) compared to participants who stated they had never been bitten (Fig. 2). The relationships between cat bites groups and non-social anticipatory and consummatory anhedonia from the TEPS were not significant. Finally, the regression with the PANSS scales found that participants reporting a first bite prior to age
13, but not after, had more severe Positive Scale symptoms (Fig. 3), when compared to participants reporting no lifetime bites. The PANSS Negative Scale and Depression severity item were not significantly related to a history of cat bites.

As all significant symptom severity findings occurred in the subgroup of participants reporting a bite prior to age 13 as compared to those who reported that they had never been bitten, we examined whether this appeared to be driven by a particular diagnostic class. As there were only 11-13 participants in the group reporting a bite prior to age 13 (depending on predictor set), quantitative examination of whether diagnostic class was a moderator was not possible. However, inspection of Figure 1 shows that for the analysis of the SPQ-BR scales, of the 13 participants who reported a cat bite prior to age 13, 4 had a schizophrenia spectrum diagnosis, 4 had a bipolar disorder diagnosis, 1 had unipolar depression, and 4 had no psychiatric diagnosis. For the anhedonia predictors (Fig. 2), 1 participant with a bipolar disorder was excluded as a statistical outlier and for the PANSS predictors (Fig. 3), 1 participant with a schizophrenia spectrum disorder and 1 with no disorder were excluded. Therefore, there did not appear to be a pattern of a particular diagnostic class driving the symptom severity findings in the subgroup of participants reporting a cat bite prior to age 13 , suggesting a relatively transdiagnostic relationship with symptom severity. 
Fig. 2. Cat bite history in relation to selfreported social anhedonia severity. Note: lower scores on the ACIPS represent greater anhedonia severity.
Fig. 3. Cat bite history in relation to clinician-rated positive symptom severity (i.e., psychotic-like experiences).
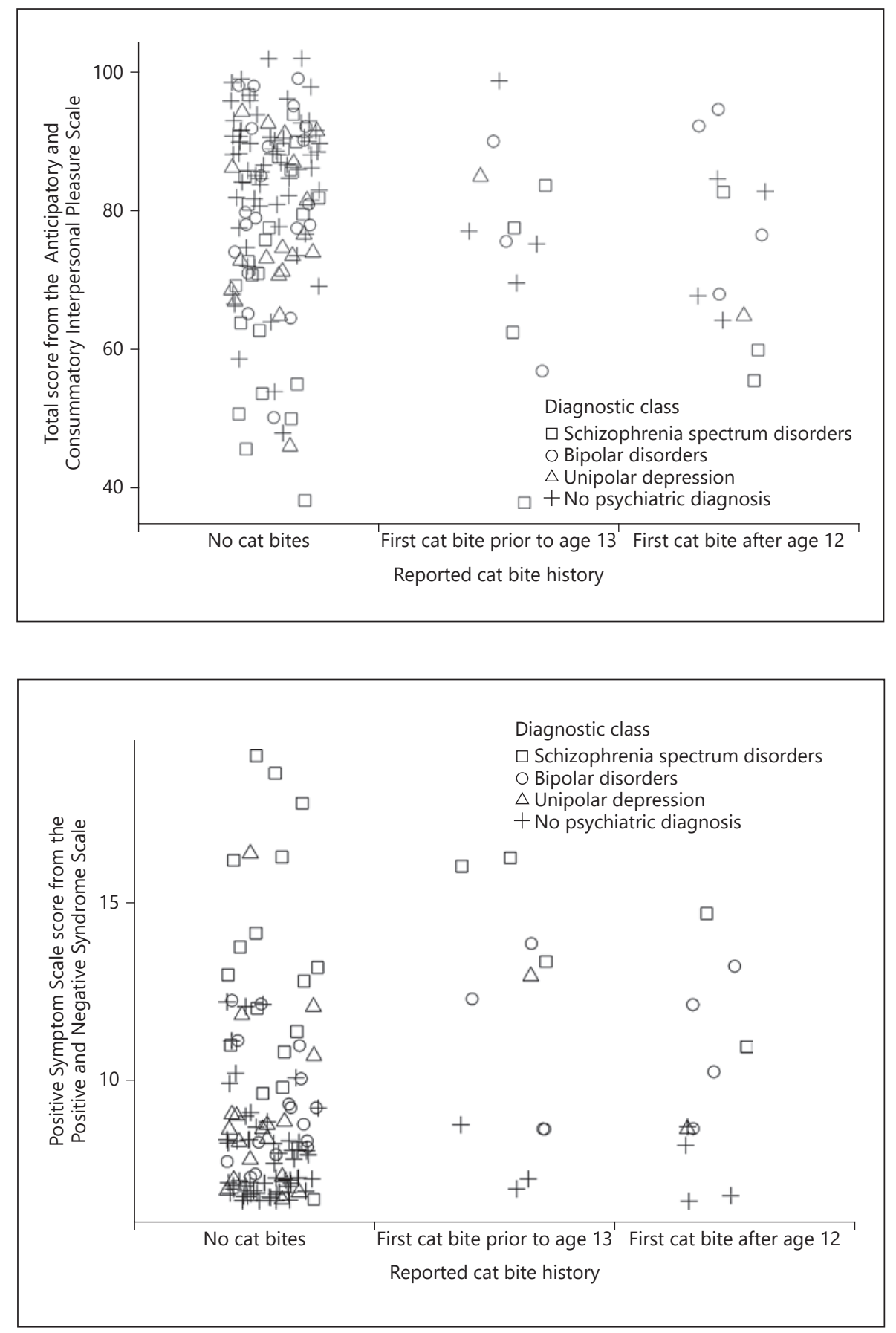

\section{Relationships with Residing with a Cat during}

\section{Childhood}

See Table 3 for descriptive and inferential statistics for all analyses involving residing with a cat prior to age 13 . Residing with a cat during childhood did not show a statistically significant relationship with any predictor variable.

\section{Discussion}

Our first hypothesis regarding a history of cat bites prior to age 13 was partially supported as participants reporting a bite prior to age 13 , but not after, had a higher total score on the SPQ-BR (i.e., overall schizotypy). This replicated our earlier findings from a nonpsychiat- 
Table 3. Descriptive statistics and binary logistic regression results related to residing with a cat in childhood

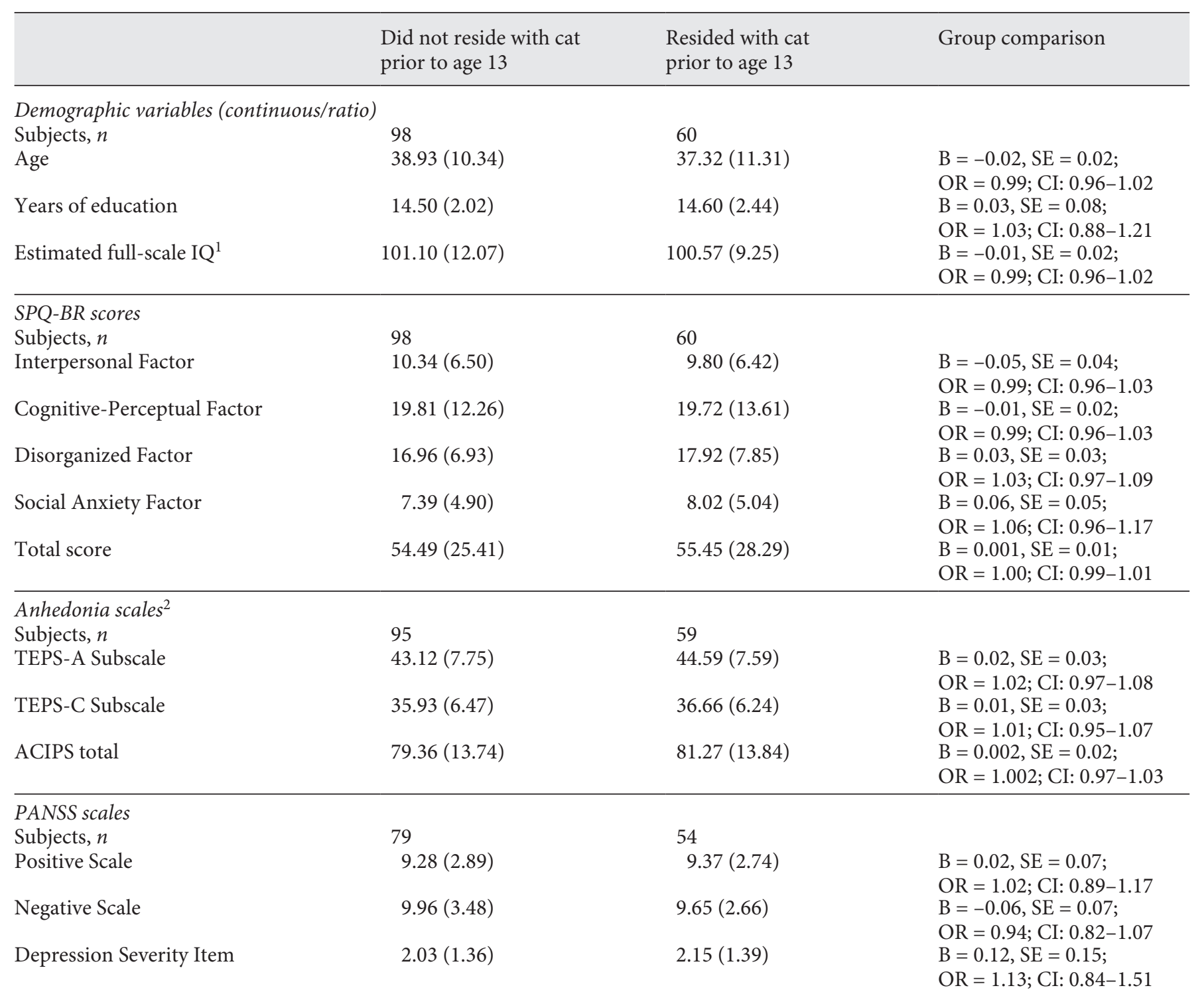

Cell values in first two columns are listed in the format: mean (standard deviation). Four of the 162 participants did not have information on residing with a cat. B, unstandardized beta; SE, standard error for beta; OR, odds ratio; CI, 95\% confidence interval for odds ratio. ${ }^{*} p<0.05 ;{ }^{* *} p<0.01,{ }^{* * *} p<0.001$ in comparison with participants reporting not residing with a cat prior to age 13 . SPQBR, Schizotypal Personality Questionnaire - Brief Revised; TEPS-A, Anticipatory subscale of the Temporal Experience of Pleasure Scale; TEPS-C, Consummatory subscale of the Temporal Experience of Pleasure Scale; ACIPS, Total score from the Anticipatory and Consummatory Interpersonal Pleasure Scale; PANSS, Positive and Negative Syndrome Scale. ${ }^{1}$ Full scale IQ estimated using the standardized score from the Reading Subtest of the Wide Range Achievement Test -4 th edition. ${ }^{2}$ For all anhedonia scales, lower scores indicate greater anhedonia (i.e., less pleasure).

ric sample [1] and extended the finding to include a transdiagnostic psychiatric sample. While we hypothesized that the Disorganized factor would show the largest effect size based on the earlier study, the current findings showed that when all four SPQ-BR factors were entered into the same regression, only the Cognitive-
Perceptual factor score was statistically significant, which was in the same direction and pattern as the total score. It is possible that the current use of transdiagnostic sample included higher scores on the Cognitive-Perceptual factor in particular, which then led to the observed relationship. 
Although not hypothesized, we found that the clinician-rated PANSS Positive Scale also was higher in those reporting a cat bite prior to age 13 , but not after, when compared to those who reported never being bitten. As the PANSS Positive Scale and the SPQ-BR CognitivePerceptual scale both assess psychotic-like experiences (e.g., hallucinatory experiences, delusions/magical thinking), the results show converging findings across both self-report and clinician-administered measures that reporting a cat bite prior to age 13 appears to relate to greater severity of psychotic-like experiences across a transdiagnostic psychiatric sample. We are not aware of any publications that examined psychotic-like experiences in relation to cat bites other than our initial publication in a nonpsychiatric sample [1]; however, one study reported no relationship between cat scratches and the adult diagnosis of schizophrenia [4], which includes psychotic symptoms. However, the earlier study [4] only assessed lifetime cat scratches, and the overlap and specificity of potential pathogens that can be transmitted from cats to humans through a cat scratch as compared to a bite (e.g., unique to saliva) remains unclear.

Our second hypothesis that a lifetime of reported cat bites would predict increased severity of depression was not supported. The omnibus test across the three cat bite groups was not statistically significant for the PANSS Depression item score, nor was the pairwise comparison of each bite age group to participants who reported no lifetime cat bites. Consistent with this clinician-rated level of recent depression severity, inspection of Figure 3 shows that unipolar depression was diagnosed in only 1 of the 11 participants in each of the two bite age groups with usable PANSS data. Our finding of a lack of relationship with depression is inconsistent with one study that reported a positive association between electronic health record diagnoses of cat bites and depression in an adult sample [5]. However, it is consistent with another study that examined both cat scratches and bites in the same model and found that only scratches related to depression [3]. In addition, the one study reporting a significant relationship with bites only assessed cat bites occurring after age 18 [5]. Although we included a category for bites occurring after age 12 , this combined bites occurring during adolescence and adulthood unlike the previous study. Finally, we only had 11 participants with usable depression severity data from the PANSS in each bite age group, providing limited statistical power, while the previous study was based on health records of over one million individuals.
Our last hypothesis was not supported, as individuals reporting that they resided with a cat prior to age $13 \mathrm{did}$ not differ on any symptom severity predictor examined, including the hypothesized relationship with increased social anhedonia (i.e., ACIPS total score). This hypothesis was based on a single study which found that selfreport of cat ownership prior to age 7 was associated with higher adult social, but not physical, anhedonia severity [11]. The previous study also reported that the relationship was found only for participants residing in a rural location with the cat, while our participants, at least in adulthood, were primarily residing in an urban/ suburban environment. Additionally, our study used a later age cutoff (age 13 vs. 7). However, partially consistent with that study [11], we also found a lack of relationship with childhood cat ownership and positive symptom severity (i.e., perceptual aberrations in the earlier study). Although exploratory, we did find that social (i.e., ACIPS total score), but not non-social (i.e., TEPS-A and TEPS-C), anhedonia was greater in participants who reported a cat bite prior to age 13, but not after, as compared to participants denying a lifetime cat bite (Fig. 2). It is possible that the finding in the earlier study [11] would have also shown a relationship with childhood cat bites that are presumably more likely in those who reside with a cat, but the study did not report data on cat bites or scratches.

For all findings involving schizophrenia or schizotypy and cat bites, the relationships were only significant for bites that were self-reported to occur prior to the age of 13 , and we only inquired about bites that punctured the skin. One theory for these findings is that an unknown infectious agent common to cat saliva interacted with brain development in childhood to increase or cause subclinical or clinical positive symptoms of schizophrenia that began or continued in adulthood. It is possible that the infectious agent is more prominent in the cat saliva rather than on the claws, as a previous study found that intensity of lifetime cat scratches did not relate to schizophrenia in adulthood [4]. However, as that study did not separately analyze scratches prior to age 13 , it is unknown whether the authors would have found a significant relationship in that age range. Although we did not examine relationships with particular diagnoses due small sample sizes of each, Figures 1-3 show that the subgroup reporting a cat bite prior to age 13 represented a similar number of participants from each of the diagnostic classes. Therefore, the symptom severity relationships we found with cat bites prior to age 13 appear to be relatively transdiagnostic. 
Several studies have implicated Toxoplasma gondii as the infectious causal agent that accounts for general relationships between cats and schizophrenia. However, one study found that the link between Toxoplasma gondii and schizophrenia was no longer significant after accounting for childhood cat ownership [7]. A possible contributing factor for the differences in findings is that some cats primarily reside inside versus outside and are therefore exposed to different pathogens based on ability to roam outside. It appears that only one published study [5] included a related moderating variable, which found that the women bitten by their own cat had a $42.9 \%$ probability of depression as compared to an only slightly higher $47.3 \%$ probability if bitten by a stray or feral cat. In contrast, for men, the probability of depression was $29.5 \%$ if bitten by their own cat which dropped notably to $12.0 \%$ if bitten by a stray of feral cat. While this study did not have data regarding whether a participant's own cat resides primarily indoors or outdoors, it provides preliminary information about an interaction of sex and risk of depression if bitten by a cat that was primarily free to roam outside.

A recent case study suggested a candidate infectious mechanism causal agent for schizophrenia may be the bacterium responsible for cat scratch disease (i.e., "cat scratch fever") - Bartonella henselae [23]. This study linked $B$. henselae seropositivity and a history of cat bites and scratches to onset of schizophrenia symptoms in a 14-year-old boy. Interestingly, the schizophrenia symptoms resolved after treatment of $B$. henselae with antimicrobial chemotherapy. Another study on 250 healthy adults found complex interactions between biological sex, Bartonella seropositivity, and Toxoplasma seropositivity in relation to depression [24]. The study concluded that Bartonella is not a likely causal infectious agent that explains the link between cat-related injuries and depression but did not examine schizophrenia-related symptoms.

Although we are not aware of others implicating the bacterium Pasteurella multocida (P. multocida), our literature review revealed that it is common in infections resulting from cat bites (75\%; compared to $50 \%$ of dog bites [25]). P. multocida causes meningitis plus neurological complications in 17-29\% of infected humans, including at the neonatal stage [25]. This bacterium has also been linked to other neurological conditions in case studies, including Miller Fisher syndrome (a form of Guillain-Barre syndrome [26]) and acute disseminated encephalomyelitis (associated with generalized brain inflammation [27]). As P. multocida is highly prevalent in

Childhood Cat Bites and Adult

Psychiatric Symptoms cat bites and possesses the ability to cause inflammation and changes to the brain, including during early development, it represents a candidate infectious mechanism that could explain the link between childhood cat bites and the observed links to psychiatric symptom severity in adulthood. Future research can directly examine this possibility. As of August 24, 2019, the publicly available Enhanced Infectious Disease (EID2) database indicates that cats (i.e., Felis catus) are known to carry 795 potential infectious agents, and of those, 197 (25\%) can also be carried by humans (https://eid2.liverpool.ac.uk/; see Acknowledgements section for details). Therefore, there are many potential infectious agents that may explain the relationship between cat-human contact and psychiatric symptoms, and very few have been examined thus far as potential causal agents. However, a study of infected cat bites in humans found a median of 6 and a maximum of 13 bacterial isolates per bite, suggesting that the number of potential infectious agents that mediate phenomena specific to cat bites rather than general cat contact, may be much smaller [28].

The study is limited by a cross-sectional design that requires participants to retroactively recall information about interactions with cats and related ages. As longterm memory is subject to distortion and the cross-sectional design cannot address causality, longitudinal studies are needed to clarify these relationships. It is also possible that individuals with higher levels of psychoticlike experiences and/or schizotypy may be more likely to have false memories of childhood cat bites. However, this explanation appears unlikely when considering that these relationships did not appear with bites reported to occur after age 12 or with having a cat in the household prior to age 13. The study is also limited by small samples of particular disorders. However, this study appears to be the first to examine relationships between cat bites and ownership in relation to severity of a variety of psychiatric symptoms in a transdiagnostic psychiatric sample. We found that the severity of overall schizotypy, self-reported and clinician-rated psychotic-like experiences, and social anhedonia were higher in adult participants who self-reported having a cat bite prior to age 13 that broke the skin, as compared to participants reporting no lifetime cat bite. There were no symptom severity relationships in those reporting a first cat bite that occurred after age 12. The current findings, along with our earlier-related schizotypy severity finding in nonpsychiatric undergraduate students [1], suggests that these relationships are relatively transdiagnostic and at least some of the symptom severity relationships can oc- 
cur in nonpsychiatric adults. Future research can examine candidate infectious agents common in cat saliva, such as $B$. henselae, $P$. multocida, and T. gondii, to identify potential causal mechanisms for these relationships. It would also be helpful to future studies to examine the sensitivity and specificity of using age 13 as a cut point for examining these relationships and to inquire about ability of the cat to roam outside. Finally, it would be useful for future studies to ask about injuries from other animals and clarify the relationship of cat scratches, as compared to bites, with a wider range of psychiatric symptoms in the same sample.

\section{Acknowledgements}

The authors would like to thank the following research assistants for assisting with data collection, data entry, and participant recruitment for this study: Dr. Chi C. Chan, Julian Montaquila, Benjamin Trachik, Dana Braden, Stanley Desire, Carina Viegas, Travis Hatfield, Nicholas Joseph, Luke Van de Krol, Adam Benzekri, Cierra Godwin, Alyssa Finner, Soraya Allen, Maya Rose, Breanna Davis, and Thomas Giallella. We acknowledge use of the EID2 database funded primarily by the European Union via the ERA ENV-HEALTH programme (ENHanCE project) and FP7 QWeCI and ICONZ projects.

\section{Statement of Ethics}

All participants provided informed consent after reviewing a written consent statement. The study was approved by the Institutional Review Board of the University of Central Florida.

\section{Disclosure Statement}

The authors have no conflicts of interest to declare.

\section{Funding Sources}

This data was funded as part of a larger study by the National Institute of Mental Health (1R15MH097222-01A1; PI: J.S. Bedwell) and matching funds from University of Central Florida College of Sciences and Office of Research and Commercialization.

\section{Author Contributions}

Author J.S.B. conceptualized the study, supervised the assignment of diagnoses, conducted the statistical analysis, and wrote the first draft of the manuscript. Author C.C.S. conducted the clinical interviews, assisted with data analysis and creation of figures, and calculated the overlap of cargo infectious agents between cats and humans using the EID2 database. Author A.L.S. assisted with presentation of statistical results and tables, and with conceptualization of theories regarding infectious agents. All authors assisted with review and editing of the final manuscript.

\section{References}

1 Kolpakova J, Bedwell JS. Childhood cat bites and disorganized symptoms of schizotypy in adulthood. Schizophr Res. 2013 May; 146(13):370-1.

2 Cohen AS, Matthews RA, Najolia GM, Brown LA. Toward a more psychometrically sound brief measure of schizotypal traits: introducing the SPQ-Brief Revised. J Pers Disord. 2010 Aug;24(4):516-37.

3 Flegr J, Hodný Z. Cat scratches, not bites, are associated with unipolar depression-crosssectional study. Parasit Vectors. 2016 Jan; 9(1):8.

4 Flegr J, Vedralova M. Specificity and nature of the associations of twenty-four neuropsychiatric disorders with contacts with cats and dogs. Schizophr Res. 2017 Nov; 189:219-20.

5 Hanauer DA, Ramakrishnan N, Seyfried LS. Describing the relationship between cat bites and human depression using data from an electronic health record. PLoS One. 2013 Aug;8(8):e70585.

6 Fuller Torrey E, Simmons W, Yolken RH. Is childhood cat ownership a risk factor for schizophrenia later in life? Schizophr Res. 2015 Jun;165(1):1-2.
7 Yuksel P, Alpay N, Babur C, Bayar R, Saribas $\mathrm{S}$, Karakose AR, et al. The role of latent toxoplasmosis in the aetiopathogenesis of schizophrenia - the risk factor or an indication of a contact with cat? Folia Parasitol (Praha). 2010 Jun;57(2):121-8.

8 Lindgren $M$, Torniainen-Holm M, Härkänen T, Dickerson F, Yolken RH, Suvisaari J. The association between toxoplasma and the psychosis continuum in a general population setting. Schizophr Res. 2018 Mar;193:329-35.

9 Burgdorf KS, Trabjerg BB, Pedersen MG, Nissen J, Banasik K, Pedersen OB, et al. Largescale study of Toxoplasma and Cytomegalovirus shows an association between infection and serious psychiatric disorders. Brain Behav Immun. 2019 Jul;79:152-8.

10 Solmi F, Hayes JF, Lewis G, Kirkbride JB. Curiosity killed the cat: no evidence of an association between cat ownership and psychotic symptoms at ages 13 and 18 years in a UK general population cohort. Psychol Med. 2017 Jul;47(9):1659-67.

11 Palomäki J, Koskela J, Suvisaari J, Yolken R, Torrey F, Veijola J. Cat ownership in childhood and development of schizophrenia. Schizophr Res. 2019 Apr;206:444-5.
12 Morris SE, Cuthbert BN. Research Domain Criteria: cognitive systems, neural circuits, and dimensions of behavior. Dialogues Clin Neurosci. 2012 Mar;14(1):29-37.

13 Bedwell JS, Spencer CC, O’Donnell JP. Promise and challenges with the research domain criteria framework. In: Beidel DC, Frueh C, editors. Adult Psychopathology and Diagnosis. 8th ed. Hoboken: John Wiley \& Sons, Inc.

14 Bedwell JS, Spencer CC, Chan CC, Butler PD, Sehatpour P, Schmidt J. The P1 visual-evoked potential, red light, and transdiagnostic psychiatric symptoms. Brain Res. 2018 May; 1687:144-54.

15 Wilkinson GS, Robertson GJ. WRAT 4: wide range achievement test. 4 th ed. Lutz (FL): Psychological Assessment Resources, Inc.; 2006.

16 Callaway DA, Cohen AS, Matthews RA, Dinzeo T. Schizotypal Personality Questionnaire-Brief Revised: psychometric replication and extension. Pers Disord. 2014 Jan;5(1):328.

17 Gard DE, Kring AM, Gard MG, Horan WP, Green MF. Anhedonia in schizophrenia: distinctions between anticipatory and consummatory pleasure. Schizophr Res. $2007 \mathrm{Jul}$; 93(1-3):253-60. 
18 Gooding DC, Pflum MJ. The assessment of interpersonal pleasure: introduction of the Anticipatory and Consummatory Interpersonal Pleasure Scale (ACIPS) and preliminary findings. Psychiatry Res. 2014 Jan;215(1): 237-43.

19 First MB, Spitzer RL, Gibbon M, Williams JB. Structured Clinical Interview for DSM-IV Axis I Disorders - CV. Washington (D.C.): American Psychiatric Press; 1997.

20 First MB, Gibbon M, Spitzer RL, Williams JB, Benjamin LS. Structured Clinical Interview for DSM-IV Axis II Personality Disorders (SCID-II). Washington (DC): American Psychiatric Press; 1997.
21 Kay SR, Opler LA, Fiszbein A. Positive and Negative Syndrome Scale (PANSS) Technical Manual. Toronto: Multi-Health Systems, Inc.; 2006.

22 Opler LA, Kay SR, Lindenmayer JP, Fiszbein A. Structured Clinical Interview for the Positive and Negative Syndrome Scale. Toronto: Multi-Health Systems, Inc.; 1999.

23 Breitschwerdt EB, Greenberg R, Maggi RG, Mozayeni BR, Lewis A, Bradley JM. Bartonella henselae bloodstream infection in a boy with pediatric acute-onset neuropsychiatric syndrome. J Cent Nerv Syst Dis. 2019 Mar; 11: 1179573519832014.

24 Flegr J, Preiss M, Balátová P. Depressiveness and neuroticism in Bartonella seropositive and seronegative subjects-preregistered casecontrols study. Front Psychiatry. 2018 Jul;9: 314.
25 Wilson BA, Ho M. Pasteurella multocida: from zoonosis to cellular microbiology. Clin Microbiol Rev. 2013 Jul;26(3):631-55.

26 Bennetto LP, Lyons P. Miller Fisher syndrome associated with Pasteurella multocida infection. J Neurol Neurosurg Psychiatry. 2004 Dec;75(12):1786-7.

27 Proulx NL, Freedman MS, Chan JW, Toye B, Code CC. Acute disseminated encephalomyelitis associated with Pasteurella multocida meningitis. Can J Neurol Sci. 2003 May;30(2): 155-8.

28 Talan DA, Citron DM, Abrahamian FM, Moran GJ, Goldstein EJ; Emergency Medicine Animal Bite Infection Study Group. Bacteriologic analysis of infected dog and cat bites. $\mathrm{N}$ Engl J Med. 1999 Jan;340(2):85-92. 